\title{
Blind Polynomial Channel Estimation for OFDM Systems
}

\author{
Y. H. Zhang, W.-S. Lu, and T. A. Gulliver \\ Department of Electrical and Computer Engineering, University of Victoria \\ P.O. Box 3055, Victoria, B.C., Canada V8W 3P6 \\ Email: yhzhang, wslu, agullive@ece.uvic.ca
}

\begin{abstract}
Orthogonal frequency division multiplexing (OFDM) modulation is widely used in communication systems to meet the demand for ever increasing data rates. Characteristics of the transmitted signal can be employed for blind channel identification. In this paper, we propose a blind polynomial channel estimation algorithm using noncircular second-order statistics of the received OFDM signal. A set of polynomial equations are then formulated based on the correlation of the received signal. The solution of these equations provides an estimate of the channel coefficients. Results are presented which show that the proposed algorithm provides performance comparable to the least minimum mean square error (LMMSE) solution with lower computational complexity. The performance is near-optimal for large OFDM systems.
\end{abstract}

\section{INTRODUCTION}

Orthogonal frequency division multiplexing (OFDM) modulation is widely employed for high data rate communications. In an OFDM system, the data stream is divided into a number of parallel lower-rate data streams, which are modulated onto orthogonal subcarriers. Thus, a frequency selective channel can be divided into a set of flat fading channels. OFDM is robust over multipath fading channels and results in significantly reduced receiver complexity [1]. The standards employing OFDM modulation include digital video broadcasting (DVB) [2], digital audio broadcasting (DAB) [3], IEEE 802.11a and 802.11g [4] for wireless local area networks, and IEEE 802.16 [5] for wireless metropolitan area networks.

For coherent detection of OFDM symbols, the receiver requires reliable channel information. Channel information can be estimated by utilizing pilot symbols in the frequency or time domains [6], but this reduces system efficiency. Alternatively, differential modulation can be implemented at the price of performance loss.

Recently, blind channel identification and equalization for OFDM system have attracted significant attention [7]-[11]. These methods estimate the channel information by exploiting the statistical behavior of the channel or received signals. Various blind estimation algorithms have been developed for OFDM systems. Chang et. al [7] employed a regression model to develop a joint blind detector for OFDM systems over timevarying channels. Cui and Tellambura [8] developed joint blind and semiblind maximum likelihood (ML) detectors which exploit the relationship between subcarriers. In [9], Heath and
Giannakis exploited the cyclostationarity introduced by the cyclic prefix (CP) to identify the channel impulse response. Cai and Akansu [10] presented a noise subspace method by utilizing the $\mathrm{CP}$ which results in faster convergence with smaller data blocks. Furthermore, Li and Roy [11] proposed a method to estimate the channel information based on virtual carriers.

Based on the source characteristics, such as the noncircularity of the input signals, blind identification techniques can be used to obtain an estimate of the channel coefficients [12]. In this paper, we take advantage of the nonzero cyclostationary statistics of the transmitted signals, which in turn allows blind polynomial channel estimation using second-order statistics of the OFDM symbol. A set of polynomial equations are formulated based on the correlation of the received signal. An estimate of the time domain channel coefficients can then be easily computed by solving these equations.

\section{SySTEM MODEL}

In an OFDM system, the bandwidth is divided into $N$ subchannels, and the data stream is modulated on the subcarriers using quadrature amplitude modulation (QAM) or phase-shift keying (PSK). The transmitted signals are generated using an inverse fast Fourier transform (IFFT)

$$
x_{n}=\frac{1}{\sqrt{N}} \sum_{k=0}^{N-1} X_{k} \exp \left(\frac{j 2 \pi k n}{N}\right) \text { for } n=0, \ldots N-1
$$

where $x_{n}$ is the time-domain signal at the $n$th sampling instant, and $X_{k}$ is the frequency-domain data symbol for the $k$ th subcarrier. Equation (1) can be written in vector form as

$$
\mathbf{X}=\mathbf{F X}
$$

where $\mathbf{x}=\left[\begin{array}{llll}x_{0} & x_{1} & \ldots & x_{N-1}\end{array}\right]^{T}$ and $\mathbf{X}=\left[\begin{array}{llll}X_{0} & X_{1} & \ldots & X_{N-1}\end{array}\right]^{T}$ represent the time-domain and frequency-domain OFDM symbols, respectively, and $T$ denotes transpose. $\mathbf{F}$ is the IFFT matrix with elements $f_{n, k}=\frac{1}{\sqrt{N}} \exp \left(\frac{j 2 \pi k n}{N}\right)$. The OFDM symbol duration is denoted by $T_{s}$, so the chip duration of each subchannel is $T_{c}=T_{s} / N$.

In this paper, we consider a frequency selective fading channel model [14], where the channel is time-invariant within 
one OFDM symbol. Thus, we have a wide sense stationary uncorrelated scattering (WSSUS) channel with impulse response given by

$$
h(\tau)=\sum_{d=1}^{D} h\left(\tau_{d}\right) \delta\left(\tau-\tau_{d}\right)
$$

where $\tau_{d}$ is the $d$ th path delay with $\tau_{1}<\tau_{2}<\ldots<\tau_{D}$. In (3), $h\left(\tau_{d}\right)$ is a complex Gaussian process with zero mean and variance $\sigma_{d}^{2} \triangleq \phi_{\tau}\left(\tau_{d}\right)$.

A discrete version of the WSSUS channel in (3) can be modeled as a tapped delay line (TDL) with channel coefficients [14]

$$
h(l)=\sum_{d=1}^{D} h\left(\tau_{d}\right) \operatorname{sinc}\left(\frac{\tau_{d}}{T_{c}}-l\right)
$$

where $h(l)$ denotes the channel coefficient for the $l$ th tap, $l=$ $0, \ldots, L-1$ with $L=\left\lfloor\tau_{D} / T_{c}\right\rfloor+1$, and the delay between two taps is $T_{c}$.

Generally a CP is inserted at the beginning of each OFDM symbol before transmission, and the length $N_{p}$ of the cyclic prefix is greater than or equal to that of the channel impulse response to eliminate the intersymbol interference. Thus, the discrete signal at the receiver can be expressed as

$$
y_{n}=\sum_{l=0}^{L-1} h(l) x(n-l)+w_{n} \text { for } n=-N_{p}, \ldots N-1
$$

where $w_{n}$ is additive white Gaussian noise (AWGN) with zero mean and variance $\sigma_{n}^{2}$. In vector form, (5) can be written as

$$
\mathbf{y}=\mathbf{H}_{\mathbf{C P}} \mathbf{x}_{\mathbf{C P}}+\mathbf{w}
$$

where $\mathbf{y}=\left[\begin{array}{llll}y_{0} & y_{1} & \ldots & y_{N-1}\end{array}\right]^{T}$ and $\mathbf{w}=$ $\left[\begin{array}{llll}w_{0} & w_{1} & \ldots & w_{N-1}\end{array}\right]^{T}$ denote the time-domain received signal and AWGN noise, respectively, and $\mathbf{x}_{\mathbf{C P}}=\left[\begin{array}{lllllll}x_{N-L+1} & x_{N-L+2} & \ldots & x_{0} & x_{1} & \ldots & x_{N-1}\end{array}\right]^{T}$. Since the CP is only a copy of part of the OFDM symbol, (6) can be written as

$$
\mathbf{y}=\mathbf{H x}+\mathbf{w}
$$

where $\mathbf{H}$ is the channel matrix given by

$$
\mathbf{H}=\left[\begin{array}{cccc}
h(0) & 0 & \ldots & h(1) \\
h(1) & h(0) & \ldots & h(2) \\
\vdots & \vdots & \ddots & \vdots \\
h(L-1) & h(L-2) & \ldots & 0 \\
\vdots & \vdots & \ddots & \vdots \\
0 & 0 & \ldots & h(0)
\end{array}\right]
$$

After removing the $\mathrm{CP}$ and performing a fast Fourier transform (FFT), we obtain

$$
\mathbf{Y}=\mathbf{A X}+\mathbf{W}
$$

where $\mathbf{Y}=\left[\begin{array}{lll}Y_{0} & \ldots Y_{N-1}\end{array}\right]^{T}$ is the frequency-domain received signal, $\mathbf{A}=\mathbf{F}^{H} \mathbf{H F}, \mathbf{W}=\mathbf{F}^{H} \mathbf{w}$, and $H$ denotes Hermitian transpose. For convenience, let $\mathbf{h}=[h(0) h(1) \ldots h(L-1)]^{T}$.

\section{Blind Polynomial Channel Estimation for OFDM SYSTEMS}

The concept of circularity was introduced in [13]. It is a simple expression of distribution, moments, and related statistical objects of the random variable, which in turn induces a correlation between the real and imaginary parts of the random variable [13]. A scalar random variable $Z$ is referred to as circular at order 2 if $E\left(Z^{2}\right)=0$, and noncircular at order 2 if $E\left(Z^{2}\right)$ is nonzero. A non-Gaussian random variable is referred as circular if its distribution is invariant when multiplied by a unit modulus complex number [12].

In this paper, we utilize the statistical property that discrete signals are noncircular at given orders (at order- $k$ for $k$-PSK random variables), to develop a polynomial channel estimation method for OFDM systems. In particular, we exploit the noncircularity at order 2 of the transmitted signal [12]. For example, for BPSK modulated signals in a single carrier system, the noncircular and circular second-order correlations are given by

$$
E\left[x_{n} x_{n+m}\right]=\delta(m)
$$

where $\delta(m)=1$ if $m=0$ and $\delta(m)=0$ elsewhere. Based on this property, a set of polynomial equations are derived such that the channel must satisfy

$$
E[y(n) y(n-m)]=\sum_{l=0}^{L-1} h(l) h(l+m)
$$

In what follows, BPSK modulation is employed in the OFDM system. Assume the transmitted signals $X_{k}$ are i.i.d. and follow a uniform distribution. The channel is timeinvariant within one OFDM symbol, and the receiver has knowledge of the channel length. Due to the noncircular statistics of the transmitted signal $X_{k}$, we have $\mathbf{R}=E\left[\mathbf{X X}^{T}\right]$ with $R_{i, j}=\delta(i-j)$. Consequently, the correlation of the time domain signal $\mathbf{x}$ is

$$
E\left[\mathbf{x x}^{T}\right]=\mathbf{F} \mathbf{F}^{T}
$$

where

$$
E[x(m) x(n)]=\frac{1}{N} \sum_{k=0}^{N-1} e^{\frac{j 2 \pi k(m+n)}{N}}=\mathbf{F}_{m}^{T} \mathbf{F}_{n}
$$

and $\mathbf{F}_{m}$ is the $m$ th column of matrix $\mathbf{F}$. Thus, the correlation $E\left[\mathbf{x x}^{T}\right]$ in (12) has the following structure

$$
E\left[\mathbf{x x}^{T}\right]=\left[\begin{array}{ccccc}
1 & 0 & 0 & \ldots & 0 \\
0 & 0 & 0 & \ldots & 1 \\
\vdots & \vdots & \vdots & \ddots & \vdots \\
0 & 0 & 1 & \ldots & 0 \\
0 & 1 & 0 & \ldots & 0
\end{array}\right]
$$

The noise and the transmitted signal are independent, and the real and imaginary parts of the noise are also independent, so we have that

$$
\begin{gathered}
E[w(n) w(n-l)]=0 \forall l \\
E\left[w(n) w(n-l)^{*}\right]=0 \text { for } l \neq 0
\end{gathered}
$$


and

$$
E\left[|w(n)|^{2}\right]=\sigma_{n}^{2}
$$

Thus, for the received signals in the time domain, the correlation is

$$
E\left[\mathbf{y} \mathbf{y}^{T}\right]=\mathbf{H F F}^{T} \mathbf{H}^{T}
$$

where

$$
E[y(n) y(n-l)]=\mathbf{F}_{n}^{T} \mathbf{h} \mathbf{h}^{T} \mathbf{F}_{n-l}
$$

Similarly, the circular second-order correlation of the transmitted signal in time domain is obtained as

$$
E\left[\mathbf{x x}^{H}\right]=\mathbf{I}_{N}
$$

As a consequence, the circular correlation of the received signals in the time domain is given by

$$
E\left[\mathbf{y} \mathbf{y}^{H}\right]=\mathbf{H H}^{H}+\sigma_{n}^{2} \mathbf{I}_{N}
$$

where

$$
E\left[y(n) y(n-l)^{*}\right]=\sum_{l=0}^{L-1} h(l)^{*} h(m+l)+\sigma_{n}^{2} \delta(l)
$$

Based on a similar concept, equivalent equations can be formulated for higher order PSK or QAM OFDM systems with some minor modifications. For such systems, the real and imaginary parts of the signals are considered separately at the receiver, because the real and imaginary parts of $X_{k}$ have a deterministic square.

\section{A. An illustrative example}

Without restricting the generality, we use a simple example to illustrate how to obtain a set of polynomial equations with respect to the channel coefficients. Assume a 6-subcarrier OFDM system with a channel of length $L=3$, then $\mathbf{h}=$ $\left[\begin{array}{lll}h_{0} & h_{1} & h_{2}\end{array}\right]^{T}$, and the channel matrix is given by

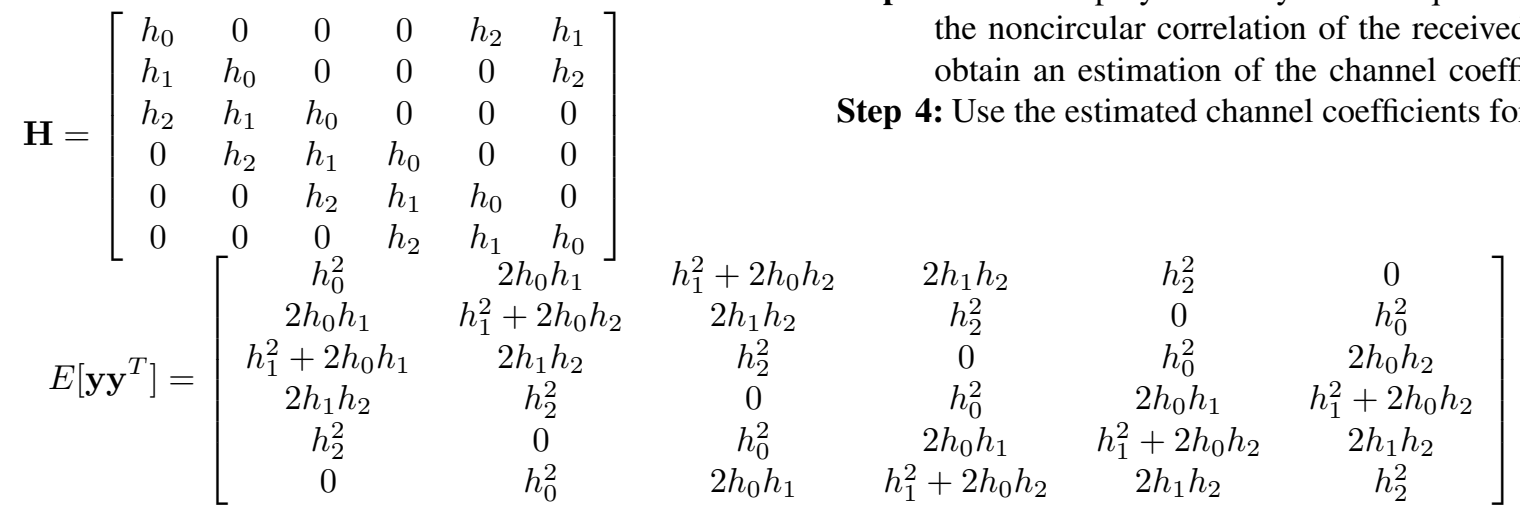

$$
\begin{aligned}
& E\left[\mathbf{y} \mathbf{y}^{H}\right]=\left[\begin{array}{cccccc}
\|\mathbf{h}\|^{2}+\sigma_{n}^{2} & h_{0} h_{1}^{*}+h_{1} h_{2}^{*} & h_{0} h_{2}^{*} & 0 & h_{2} h_{0}^{*} & h_{2} h_{1}^{*}+h_{1} h_{0}^{*} \\
h_{2} h_{1}^{*}+h_{1} h_{0}^{*} & \|\mathbf{h}\|^{2}+\sigma_{n}^{2} & h_{0} h_{1}^{*}+h_{1} h_{2}^{*} & h_{0} h_{2}^{*} & 0 & h_{2} h_{0}^{*} \\
h_{2} h_{0}^{*} & h_{2} h_{1}^{*}+h_{1} h_{0}^{*} & \|\mathbf{h}\|^{2}+\sigma_{n}^{2} & h_{0} h_{1}^{*}+h_{1} h_{2}^{*} & h_{0} h_{2}^{*} & 0 \\
0 & h_{2} h_{0}^{*} & h_{2} h_{1}^{*}+h_{1} h_{0}^{*} & \|\mathbf{h}\|^{2}+\sigma_{n}^{2} & h_{0} h_{1}^{*}+h_{1} h_{2}^{*} & h_{0} h_{2}^{*} \\
h_{0} h_{2}^{*} & 0 & h_{2} h_{0}^{*} & h_{2} h_{1}^{*}+h_{1} h_{0}^{*} & \|\mathbf{h}\|^{2}+\sigma_{n}^{2} & h_{0} h_{1}^{*}+h_{1} h_{2}^{*} \\
h_{0} h_{1}^{*}+h_{1} h_{2}^{*} & h_{0} h_{2}^{*} & 0 & h_{2} h_{0}^{*} & h_{2} h_{1}^{*}+h_{1} h_{0}^{*} & \|\mathbf{h}\|^{2}+\sigma_{n}^{2}
\end{array}\right]
\end{aligned}
$$

After removing the $\mathrm{CP}$, the noncircular and circular correlation of the received signals in the time domain have the structures given in (22) and (23), respectively. It can be seen that both $E\left[\mathbf{y} \mathbf{y}^{T}\right]$ and $E\left[\mathbf{y} \mathbf{y}^{H}\right]$ are circular matrices with respect to

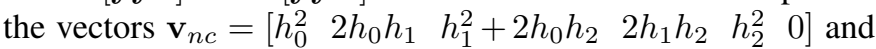
$\mathbf{v}_{c}=\left[\|\mathbf{h}\|^{2}+\sigma_{n}^{2} h_{0} h_{1}^{*}+h_{1} h_{2}^{*} h_{0} h_{2}^{*} \quad 0 \quad h_{2} h_{0}^{*} h_{2} h_{1}^{*}+h_{1} h_{0}^{*}\right]$, respectively. Both vectors have $2 L-1$ nonzero components. Thus we can obtain a system of $2 L-1$ second order polynomial equations, which corresponds to $L$ unknown channel coefficients. Note that $\mathbf{v}_{n c}$ is only related to the channel coefficients, and the polynomial system based on the noncircular correlation is easier to solve. Based on the noncircular correlation $E\left[\mathbf{y y}^{T}\right]$, the polynomial system of equations is

$$
\left\{\begin{array}{l}
f_{1}(\mathbf{h})=h_{0}^{2}-\alpha_{1}=0 \\
f_{2}(\mathbf{h})=2 h_{0} h_{1}-\alpha_{2}=0 \\
f_{3}(\mathbf{h})=h_{1}^{2}+2 h_{0} h_{3}-\alpha_{2}=0 \\
f_{4}(\mathbf{h})=2 h_{1} h_{2}-\alpha_{4}=0 \\
f_{5}(\mathbf{h})=h_{2}^{2}-\alpha_{5}=0
\end{array}\right.
$$

with $\alpha_{i}=\mathbf{v}_{n c}(i)$.

Several solutions are possible using the first, second and fifth equations in (21). The other two equations allow a unique solution of the channel coefficients, up to a sign. ¿From this example, it can be seen that it is possible to identify a complex-valued channel by using the noncircular second-order statistics or circular second order correlation using only simple computations. It is worth noting that the computational complexity of the proposed method is $O\left(N^{2}\right)$, which corresponds to the calculation of the correlation matrix $\mathbf{y} \mathbf{y}^{T}$.

We summarize the polynomial channel estimation method below.

Step 1: Obtain the received signal $\mathbf{y}$, and compute the correlation product $\mathbf{y y}^{T}$.

Step 2: Shift the rows of $\mathbf{y y}^{T}$ with respect to the first row and average them over the subcarriers to obtain an approximation of $\mathbf{v}_{n c}$.

Step 3: Solve the polynomial system of equations based on 


\section{PERFormance EVAluation}

The proposed blind polynomial channel estimation algorithm was applied to an OFDM system with BPSK modulation. The carrier frequency of the OFDM system was $5 \mathrm{GHz}$ and the bandwidth of the system was set to $200 \mathrm{kHz}$. A WSSUS channel was employed with an exponential multipath intensity profile, i.e., $\sigma_{d}^{2}=\exp (-d / D) / \sum_{d=0}^{D-1} \sigma_{d}^{2}$. The simulations were carried out for various numbers of channel taps, where each tap is an independent complex Gaussian random process with Jake's Doppler spectrum. Two pilot tones were utilized to compensate for the sign ambiguity. The channel length is assumed to be known at the receiver, and a one-tap zero forcing $(\mathrm{ZF})$ equalizer was employed to detect the received signals. The normalized Doppler frequency of the channel was set to $f_{d} T_{s}=0.001$. The performance of the proposed algorithm was evaluated based on the mean square error (MSE), bit error rate (BER) and computational complexity.

Fig. 1 shows the BER performance of a 6-tap OFDM system for different channel estimation methods with $N=128$ subcarriers. It can be observed that the blind polynomial channel estimation algorithm outperforms the least minimum mean square error (LMMSE) solution at high SNRs with similar computational complexity. For example, for the proposed method, a BER of $10^{-3}$ was achieved at $E_{b} / N_{0}=25.5 \mathrm{~dB}$, with approximately $0.5 \mathrm{~dB}$ loss compared to that with perfect channel state information (CSI). To obtain the same BER with the LMMSE method, $E_{b} / N_{0}=27 \mathrm{~dB}$ is necessary, and additional information such as the noise variance and other channel statistics are required.

Simulations were carried out to determine the impact of the channel length on the system performance. The MSE of the proposed algorithm for various channel lengths and $N=128$ is depicted in Fig. 2. It can be observed that the proposed algorithm achieves better performance with a shorter channel length. This is because $\mathbf{v}_{n c}$ has more nonzero components with a longer length channel, which results in greater computational errors. For example, the proposed algorithm achieves an MSE of $10^{-3}$ at $E_{b} / N_{0}=17 \mathrm{~dB}$ in a 3-tap channel, while the same MSE was achieved at $E_{b} / N_{0}=22 \mathrm{~dB}$ in a 5-tap channel.

The BER performance of the proposed algorithm with various values of $N$ in a 6-tap channel is shown in Fig. 3. It can be observed that the BER performance improves as the number of subcarriers increases. The reason is that the proposed algorithm solves the polynomial equations based on the approximate noncircular second order statistics. As the number of subcarriers increases, the effect of noise on the calculation $\mathbf{v}_{n c}$ from $\mathbf{y} \mathbf{y}^{T}$ is reduced. For example, with $N=64$, the proposed algorithm achieves a BER of $10^{-3}$ at $E_{b} / N_{0}=28 \mathrm{~dB}$, with approximately a $1.5 \mathrm{~dB}$ loss compared to perfect CSI. With $N=256$, the same BER can be reached at $E_{b} / N_{0}=26 \mathrm{~dB}$ with almost no loss compared to perfect CSI.

\section{CONCLusions}

Based on the source characteristics, such as the noncircular characteristics of the input signals, blind channel identification techniques can be developed to obtain a system of polynomial equations. In this paper, we exploited the nonzero cyclostationary statistics of the transmitted signal to allow blind polynomial channel estimation using the second-order statistics. A set of polynomial equations was formulated based on the correlation of the received signals. The solution of these equations was easily obtained as the channel coefficients are in the time domain. Performance results were presented which show that the proposed algorithm provides better performance than the LMMSE solution at high SNRs with low computational complexity. Near-optimal performance can be achieved with large OFDM systems.

\section{REFERENCES}

[1] R. V. Nee and R. Prasad, OFDM for Wireless Multimedia Communications, Artech House, 2000.

[2] ETSI, "Digital video broadcasting: Framing structure, channel coding, and modulation for digital terrestrial television," European Telecommunication Standard, ETS 300-744, Aug. 1997.

[3] ETSI, "Radio broadcasting systems: Digital audio broadcasting to mobile, portable and fixed receivers," European Telecommunication Standard, ETS 300-401, Feb. 1995.

[4] IEEE 802.11, "IEEE standard for wireless LAN: Medium access control (MAC) and physical layer (PHY) specifications," Nov. 1997.

[5] IEEE 802.16, "IEEE standard for local and metropolitan area networks: Air interface for fixed broadband wireless access systems," June 2004.

[6] M. K. Ozdemir and H. Arslan, "Channel estimation for wireless OFDM systems," IEEE Commun. Surveys and Tutorials, vol. 9, pp. 18-48, 2007.

[7] M.-X. Chang and Y. T. Su, "Blind and semiblind detections of OFDM signals in fading channels," IEEE Trans. Commun., vol. 52, no. 5, pp. 744-754, May. 2004.

[8] T. Cui and C. Tellambura, "Joint data detection and channel estimation for OFDM systems," IEEE Trans. Commun., vol. 54, no. 4, pp. 670-679, April 2006.

[9] R. W. Heath and G. B. Giannakis, "Exploiting input cyclostationarity for blind channel identification in OFDM systems," IEEE Trans. Signal Processing, vol. 47, no. 3, pp. 848-856, Mar. 1999.

[10] X. Cai and A. N. Akansu, "A subspace method for blind channel identification in OFDM systems," in Proc. IEEE Int. Conf. on Commun., pp. 929-933, June 2000

[11] C. Li and S. Roy, "Subspace-based blind channel estimation for OFDM by exploiting virtual carriers," IEEE Trans. Wireless Commun., vol. 2, no. 1, pp. 141-150, Jan. 2003.

[12] O. Grellier, P. Comom, B. Mourrain, and P. Trebuchet, "Analytical blind channel identification," IEEE Trans. Signal Processing, vol. 50, no. 9, pp. 2196-2207, Sept. 2002.

[13] B. Picinbono, "On circularity," IEEE Trans. Signal Processing, vol. 42, no. 12, pp. 3473-3482, Dec. 1994.

[14] J. G. Proakis, Digital Communications, 4th Ed., McGraw-Hill, 2001. 


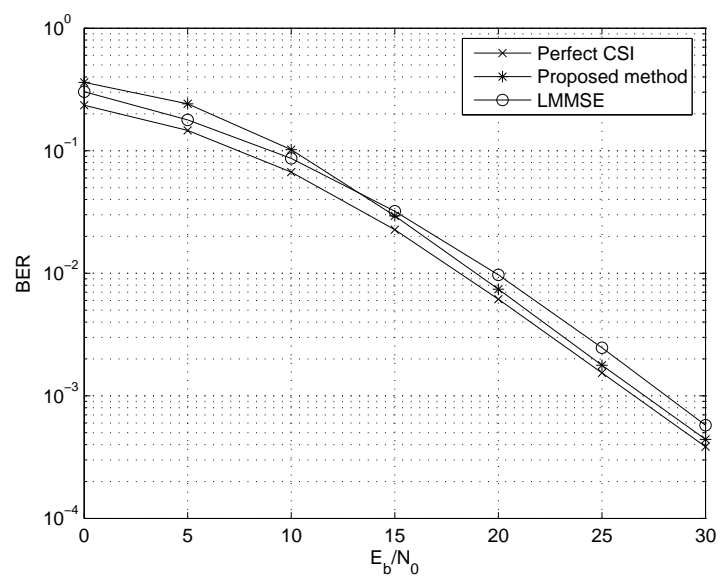

Fig. 1. BER performance of a 6-tap 128-subcarrier OFDM system.

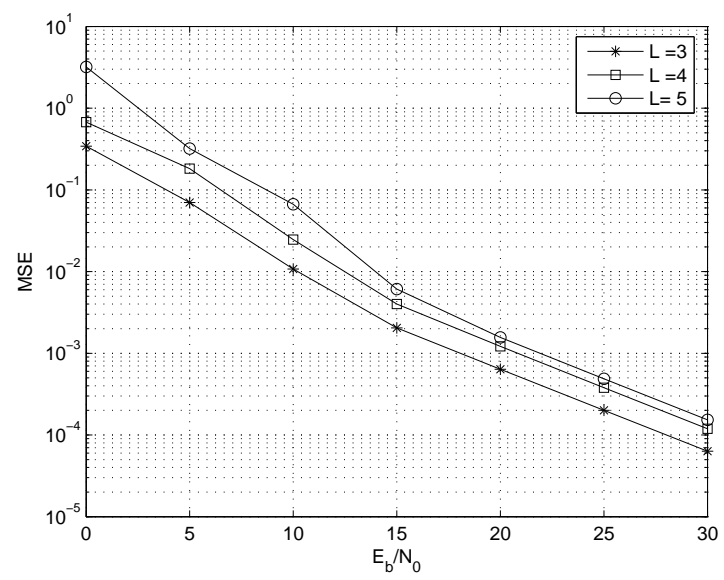

Fig. 2. Mean square error of the proposed method for a 128-subcarrier OFDM system with various channel lengths.

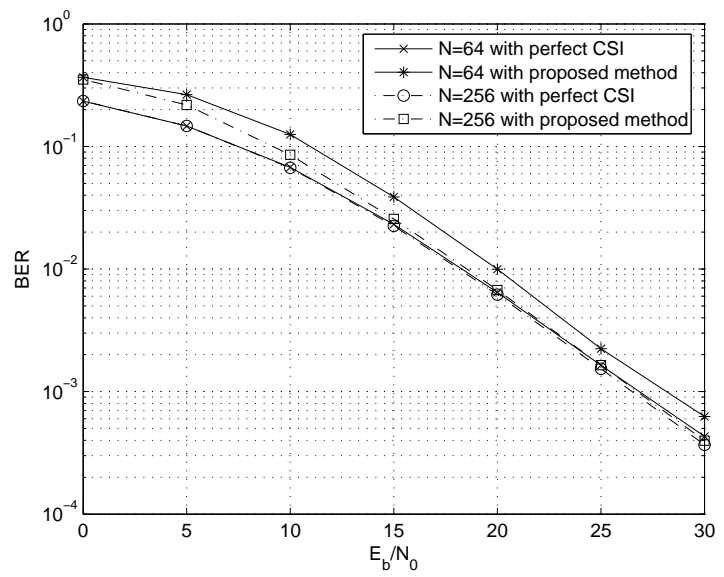

Fig. 3. BER performance of the proposed method for an OFDM system with various numbers of subcarriers and a 6-tap channel. 